\title{
MBP-hydrolyzing abzymes as a peripheral markers associated with impaired myelination in schizophrenia
}

\author{
Daria Parshukova \\ Mental Health Research Institute \\ Tomsk National Research Medical \\ Center RAS \\ Tomsk, Russia \\ Sus12008@yandex.ru \\ Arkady Semke \\ Mental Health Research Institute \\ Tomsk National Research Medical \\ Center RAS \\ Tomsk, Russia \\ Asemke@mail.ru
}

\author{
Liudmila Smirnova \\ Mental Health Research Institute \\ Tomsk National Research Medical \\ Center RAS \\ Tomsk, Russia \\ Lpsmirnova@yandex.ru \\ Vasily Yarnykh \\ University of Washington, Department \\ of Radiology, \\ Seattle WA, USA
}

\author{
Ekaterina Dmitrieva \\ Mental Health Research Institute \\ Tomsk National Research Medical \\ Center RAS \\ Tomsk, Russia \\ Egdtomsk@mail.ru \\ Svetlana Ivanova \\ Mental Health Research Institute \\ Tomsk National Research Medical \\ Center RAS \\ Tomsk, Russia \\ Ivanovaniipz@gmail.com
}

\begin{abstract}
It is well-known that the pathology of myelin and oligodendrocytes is involved in the pathogenesis of schizophrenia. Anomalies in oligodendrocytes and myelin can be a source of neuronal disruption. The discovery of catalytic antibodies (abzymes) allows us to investigate their pathological role in various conditions. One of the possible ways of inducing proteolytic antibodies is the appearance in the peripheral blood of a substrate in the form of damaged protein fragments. In our previous study, it was shown that the IgG of schizophrenia patients can hydrolyze the myelin basic protein - one of the main components of the central nervous system myelin. The study of IgG proteases in accessible biomaterial (serum) and their association with myelination disturbance in schizophrenia may be potential criteria for monitoring the severity of mental disorders.
\end{abstract} $M B P$

Keywords - myelin; abzymes; schizophrenia; neuroimaging;

\section{MOTIVATION AND AIM}

Numerous data from intravital neuroimaging, as well as genetic and morphological studies, indicate impaired myelination and pathology of oligodendrocytes in the brain of patients with schizophrenia. Post-mortem studies in schizophrenia using electron microscopy and histochemistry confirm structural changes in oligodendroglia cells producing the myelin sheath [1], atrophy of axons, abnormalities of the myelin sheath surrounding [2], reduce in myelin basic protein in the anterior frontal cortex of cases of schizophrenia [3] and an increase in the antibody titer to myelin basic protein. Recently we reported about autoantibodies against myelin basic protein (MBP) which had catalytic activity and were capable to hydrolyze myelin basic protein and its peptides [4]. The level of MBP-hydrolyzing activity in patients with schizophrenia significantly exceeded the level of activity of antibodies from healthy donors and depended on the leading symptoms and duration of the disease.

Various neuroimaging techniques reported volume abnormalities in different areas of the brain in schizophrenia. MRI protocols allowing quantification of myelin density have been developed. Macromolecular proton fraction (MPF) mapping is a quantitative MRI method that reconstructs parametric maps of a relative amount of macromolecular protons causing the magnetization transfer (MT) effect and provides a quantitative biomarker of myelin in neural tissues [5]. This study focuses on investigation the level of activity of MBP-hydrolyzing antibodies and myelin density in patients with schizophrenia.

\section{MATERIALS AND METHODS}

\section{Patients}

In this work, 15 patients with paranoid schizophrenia and 14 healthy donors of match age were recruited to study. The inclusion criteria were the following: or simple schizophrenia according with the International Statistical Classification of Diseases and Related Health Problems, 10th Revision (ICD10: F20.0). The exclusion criteria were the following: the presence of acute and chronic infectious, inflammatory, autoimmune, or neurological diseases, other organic mental disorders (e.g., epilepsy, Parkinson's disease) and mental retardation. The patients were recruited from the Mental Health Research Institute TNMRC, Department of Endogenous Disorders (Tomsk, Russia).

\section{Purification of immunoglobulins G from schizophrenia patients' serum}

Antibodies from the blood sera of schizophrenia patients and healthy controls were purified and analyzed by earlier developed procedures for purification of electrophoretically and immunologically homogenous IgG preparations from human blood serum. The procedure included affinity chromatography of serum proteins on Protein G-Sepharose followed by high-performance gel filtration on a Superdex200 HR 10/30 column.

\section{Evaluation of Proteolytic activity of serum $\operatorname{IgGS}$}

IgG of schizophrenia patient were incubated with MBP at $37 \mathrm{C}$ during 5-14 hours. The catalytic activity of the IgG in the cleavage of MBP was estimated from the decrease in the intensity of Coomassie-stained MBP band after electrophoresis. The activity of the Abs is expressed in units of specific enzyme activity as quantity $\mathrm{mg}$ of substrate cleavage by $1 \mathrm{mg}$ Abs per $1 \mathrm{~h}$. Evaluation of IgG-depended proteolytic activity was performed during the period of exacerbation of clinical symptoms.

\section{In vivo determination of myelin density by mapping}

of the molecular proton fraction using MRI

Images were acquired by using a 1.5-T imager (Magnetom Essenza; Siemens, Germany) with implication of fast threedimensional whole-brain MPF mapping protocol [5]. Statistical analyses were performed in the program Statistica 
10.0. To take into account partial volume effects, four tissue classes were prescribed within the single-channel segmentation procedure. These classes correspond to pure weight matter (WM), pure gray matter (GM), mixed voxels that contained partial volumes from WM and GM (PVWGM), and mixed voxels that contained a partial volume from cerebrospinal fluid - boundary layer (BL).

\section{RESULTS}

In this work, we revealed a statistically significant decrease in MPF in global gray matter $(p=0,002)$, mixed substance $(p=0,014)$, cortical gray matter $(p=0,001)$ in patients with schizophrenia compared with the control. In addition, a statistically significant decrease in MPF was revealed in schizophrenic patients with predominant negative symptoms compared to the control group in global gray matter $(p=0,001)$, white matter $(p=0,01)$, mixed substance $(p=0,004)$ and cortical gray matter $(\mathrm{p}<0,001)$. In our previous study, it was shown that the greatest MBP-hydrolyzing activity is associated with negative symptoms.

Since the indicators of proteolytic activity and MPF density in patients with schizophrenia showed similar changes in the analyzed groups, an analysis of the correlation relationships of these indicators was performed to assess the degree of their mutual influence. For this analysis the MRI scan and the investigation of proteolytic activity was performed in same group of patients. The results of the correlation analysis of myelin density and specific activity are shown in Table 30. Correlation analysis revealed a reliable negative correlation of average strength $(r=-0.593 ; p=0.019)$ between the level of specific activity of MBP-hydrolyzing IgG and myelin density (MPF) in the white matter of the brain.

The presented results show that catalytic antibodies with MBP-hydrolyzing activity can be used as peripheral markers which reflect a decrease in the density of myelin in the structure of the brain.

\section{ACKNOWLEDGMENT}

Support by Grant of RSF No. 18-15-00053 «Search of peripheral markers associated with impaired myelination of the brain and pathogenesis of schizophrenia» 2018-2020.

\section{REFERENCES}

[1] Martins-de-Souza D et al. (2010). Proteome and transcriptome analysis suggests oligodendrocyte dysfunction in schizophrenia. J. Psychiatr. Res. 44(3), 149-156.

[2] Uranova, N. A., Vostrikov, V. M., Orlovskaya, D. D., Rachmanova, V. I. (2004). Oligodendroglial density in the prefrontal cortex in schizophrenia and mood disorders: a study from the Stanley Neuropathology Consortium. Schizophr. Res. 67(2), 269-275.

[3] Matthews, P. R., 2012 23. Matthews, P. R., Eastwood, S. L., Harrison, P. J. (2012). Reduced myelin basic protein and actin-related gene expression in visual cortex in schizophrenia. PloS One. 7(6), e38211.

[4] Parshukova D.A. et al. (2019) Autoimmunity and immune system dysregulation in schizophrenia: IgGs from sera of patients hydrolyze myelin basic protein. J Mol Recognit. e2759 doi: 10.1002/jmr.2759

[5] Yarnykh, V. L. et al. (2016). Time - efficient, high - resolution, whole brain three - dimensional macromolecular proton fraction mapping. Magnetic resonance in medicine. 75(5), 2100-2106. 